\title{
Analysis of the Rotor Magnetomotive Force of Built-In Radial Permanent Magnet Generator for Vehicle
}

\author{
Huihui Geng, ${ }^{1}$ Xueyi Zhang $\mathbb{D}^{1}{ }^{1}$ Tao Si, ${ }^{2}$ Lanian Tong, ${ }^{2}$ Qingzhi Ma, ${ }^{2}$ and Mingjun $\mathrm{Xu}^{1}$ \\ ${ }^{1}$ School of Transportation and Vehicle Engineering, Shandong University of Technology, Zibo 255049, China \\ ${ }^{2}$ Technology Department, Shandong Tangjun Ouling Automobile Manufacture Co., Ltd., Zibo 255185, China \\ Correspondence should be addressed to Xueyi Zhang; zhangxueyi@sdut.edu.cn
}

Received 28 April 2021; Accepted 26 October 2021; Published 8 November 2021

Academic Editor: Bhupendra Khandelwal

Copyright (C) 2021 Huihui Geng et al. This is an open access article distributed under the Creative Commons Attribution License, which permits unrestricted use, distribution, and reproduction in any medium, provided the original work is properly cited.

\begin{abstract}
Permanent magnet generator (PMG) for vehicles has attracted more and more attention because of its high efficiency, high power density, and high reliability. However, the weak main air-gap magnetic field can affect the output performance and the normal use of electrical equipment. Aiming at the problem, this paper took the rotor magnetomotive force (MMF), the direct influencing parameter of the main air-gap magnetic field, as the research object, deduced the analytical expression of rotor MMF of the built-in radial PMG in detail, and analyzed its main influencing factors in analytical expression, including the permanent magnet steel (PMS) material, the thickness of PMS in magnetizing direction, the vertical length of the inner side of PMS, and the effective calculation length of PMS. Based on this, the rotor parameters were optimized to obtain the best values. After that, the finite element simulation and prototype test of the optimized generator were carried out. The comparative analysis results showed that the optimized rotor parameters could effectively improve the rotor MMF and optimize the output performance of the generator.
\end{abstract}

\section{Introduction}

The performance of the vehicle generators directly affects the performance and service life of the electrical equipment. Permanent magnet generator (PMG) has a broad application prospect because of its advantages of high efficiency, high power density, and high reliability [1-3]. Compared with the built-in tangential PMG, the permanent magnet steel (PMS) of the surface-mounted PMG and the built-in radial PMG is closer to the main air gap, and the distance between the PMSs is closer too, so there is less magnetic leakage between them. Moreover, compared with the surface-mounted PMG, the built-in radial PMG has high structural strength and high reliability and therefore has become the future development trend [4-7]. However, the rotor magnetic field of this generator is provided by two adjacent radial PMSs in series and therefore needs to pass through two PMSs. Due to the large magnetic resistance of the PMS, the rotor magnetic field is weakened, which can lead to the weakening of the air-gap magnetic field and affect the output performance of the generator [8]. Therefore, it is of great significance to accurately analyze the influencing factors of rotor magnetic field and improve the main airgap magnetic field and the output characteristics of the generator.

At present, there are two main methods to study the main air-gap magnetic field. One is the finite element method, which mainly uses the finite element software to analyze the variation law of the main structural parameters and the main air-gap magnetic field parameters, for example, the study [9] used the finite element method to analyze the air-gap magnetic flux density and its Fourier variation parameters of a permanent magnet motor with two rotors, studied the variation law of design parameters and air-gap magnetic flux density, and obtained the optimal stator parameters. In [10], the influence of the coupling effect of permanent magnet linear motor on the air-gap magnetic field was analyzed by finite element method, and the key parameters were analyzed and optimized. The other research method for the main air-gap magnetic field is the equivalent magnetic circuit method, which method analyzes the influence of different structures on the main air-gap 
magnetic field by establishing a simple equivalent model. For example, in [11], the equivalent magnetic circuit model of permanent magnet linear motor considering magnetic saturation effect was derived. The expression of main magnetic flux was calculated, and the model was verified and calibrated by experiments. Ref. [12] established an equivalent magnetic circuit model by analyzing the change of air-gap reluctance of a switched reluctance motor at different rotor positions and analyzed the output performance of the motor. This method can analyze the magnetic circuit distribution law of different topologies, but most usually use simple reluctance and magnetic conductivity as parameters, and the accuracy is limited. Even if the test method is used for correction as in [10], it cannot adapt to the motors of other sizes.

Therefore, some scholars began to study the detailed rotor magnetomotive force (MMF) model and substitute it into the equivalent magnetic circuit model to improve the accuracy. Rotor MMF is the MMF that the rotor magnetic field can provide for the main air gap. It is the main target parameter of rotor magnetic circuit design and is mainly used for the analysis of rotor magnetic field. Rotor MMF was used to calculate the main air-gap magnetic field in the early stage. Some scholars calculated the main air-gap magnetic field as the product of the rotor MMF function and the main air-gap length function [13]. Then, the rotor MMF was used to assist in calculating the electromagnetic parameters and related parameters of the generator, such as main air-gap magnetic flux, induced electromotive force and its harmonics, and mutual inductance between rotor and stator magnetic circuit. For example, Ref. [14] proposed an equivalent magnetic circuit model of a single rotor circuit of a doubly fed induction motor, took the spatial harmonic of rotor MMF as the influence parameter of rotor magnetic field quality into the model, and calculated the spatial processes of self-induction, mutual inductance, and back electromotive force of generator. Ref. [15] studied the changes of rotor MMF and its harmonics with different stacking addenda of PMS in rotor core of the built-in multilayer PMG. Then, it analyzed the stator iron loss law based on the optimization of rotor MMF. In [16], the rotor MMF of hybrid excitation salient pole motor was derived based on the principle of minimum reluctance. Based on this, the magnetic permeability function of the main magnetic field considering nonuniform air gap was calculated by using the equivalent magnetic circuit method. At present, the research on rotor MMF still focuses on the simple equivalent methods of magnetic conductivity and reluctance and less on the analytical calculation of magnetic circuit distribution [17-22]. However, the rotor structure parameters directly affect the magnitude of the rotor MMF, so it is necessary to find out the relationship between them. On this basis, this paper deduced the analytical expression of the rotor MMF of the built-in radial PMG in detail, analyzed the influencing factors in the analytical expression, and took a 3-phase 8-pole 36-slot built-in radial PMG as an example to analyze the influence rules of the rotor parameters on the rotor magnetic field and main air-gap magnetic field.
TABLE 1: The technical indicators of the generator.

\begin{tabular}{lc}
\hline Main parameters & Parameter value \\
\hline Rated power & $1000 \mathrm{~W}$ \\
Rated voltage & $14 \mathrm{~V}$ \\
Rated speed & $4000 \mathrm{r} / \mathrm{min}$ \\
Cooling mode & Self-cooling fan IC01 \\
Working temperature & $-40^{\circ} \mathrm{C} \sim 75^{\circ} \mathrm{C}$ \\
\hline
\end{tabular}

\section{Equivalent Magnetic Circuit Analysis and Analytical Expression of Rotor MMF}

Taking a 3-phase 8-pole 36-slot radial PMG as an example, its technical indicators are shown in Table 1. In this generator, the PMSs are evenly embedded in the rotor core in the radial direction, and to reduce the magnetic leakage at both ends of the PMS, magnetic separation slots are set at both ends. In addition, the rotor is laminated and welded with $0.5 \mathrm{~mm}$ silicon steel sheets in order to reduce eddy current loss. The generator structure and the rotor punching are shown in Figure 1, and the magnetic circuit of the main flux and leakage magnetic flux is shown in Figure 2.

As shown in Figure 2, the main flux of the generator passes through the rotor core of the two poles and the PMS. Then, it passes through the main air gap and the stator core to form a circuit, whereas the leakage magnetic flux mainly passes through the two ends of the PMS and forms a loop through the rotor core. According to the magnetic circuit analysis, the equivalent magnetic circuit diagram is drawn and shown in Figure 3.

In Figure 3, the magnetic field of PMS is equivalent to the series connection of permanent magnetic MMF and internal permeance. And in Figure $3, F_{\mathrm{pm} 1}$ and $F_{\mathrm{pm} 2}$ are the MMFs generated by the adjacent two pole PMS, respectively. $G_{\mathrm{pm}}$ is the internal permeance of PMS. $G_{l 1}, G_{l 2}$, and $G_{l 3}$ are, respectively, the permeance of the magnetic circuit inside the PMS of two adjacent magnetic poles, the permeance of the outer magnetic circuit of the PMS, and the leakage permeance of the outer magnetic circuit of the PMS. $G_{\delta}$ is the permeance of main air gap. $G_{\text {st }}$ is the permeance of the magnetic circuit of the stator. $\Phi_{\mathrm{pm}}$ is total magnetic flux produced by two PMS. $\Phi_{r}$ is the leakage magnetic flux at the end of PMS. $\Phi_{u}$ is the effective magnetic flux of the generator, and $F_{\text {ad }}$ is the direct axis reaction potential of the generator. Figure 3 shows that the main air-gap magnetic field is mainly related to the MMF of PMS, the internal permeance of PMS, the permeance of the magnetic circuit inside the rotor core, the permeance of the main air gap, the permeance of the magnetic circuit inside the stator, and the direct axis reaction potential of the generator. Among them, due to the application requirements of the generator, the main technical parameters are basically unchanged. That is, the permeance of the main air gap, the permeance of the magnetic circuit inside the stator, and the direct axis reaction potential of the generator are basically unchanged, and the main air-gap magnetic field is mainly related to the magnetic circuit inside the rotor core, 


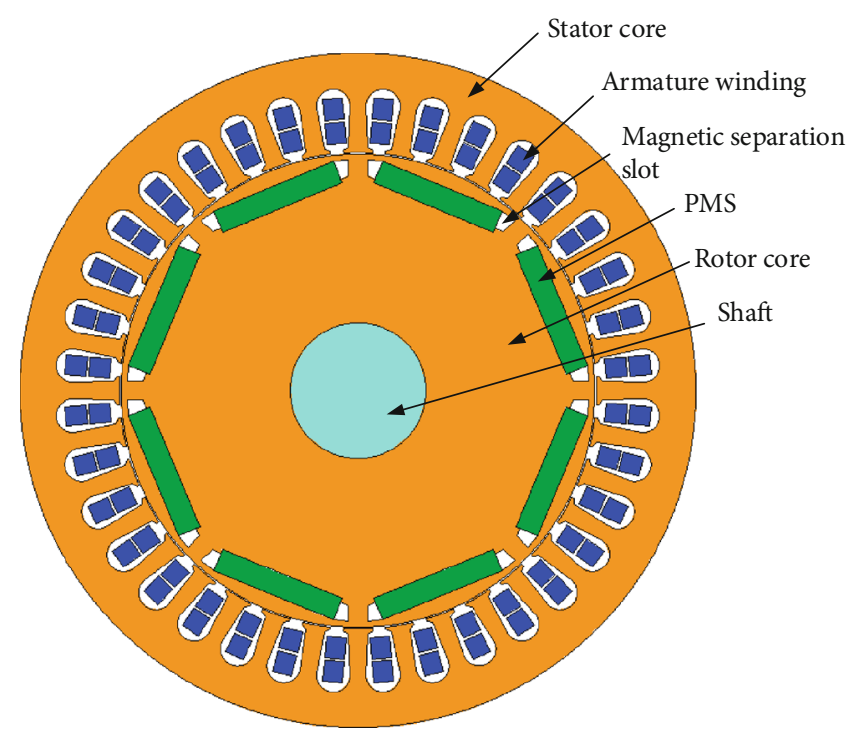

(a) The structure of PMG

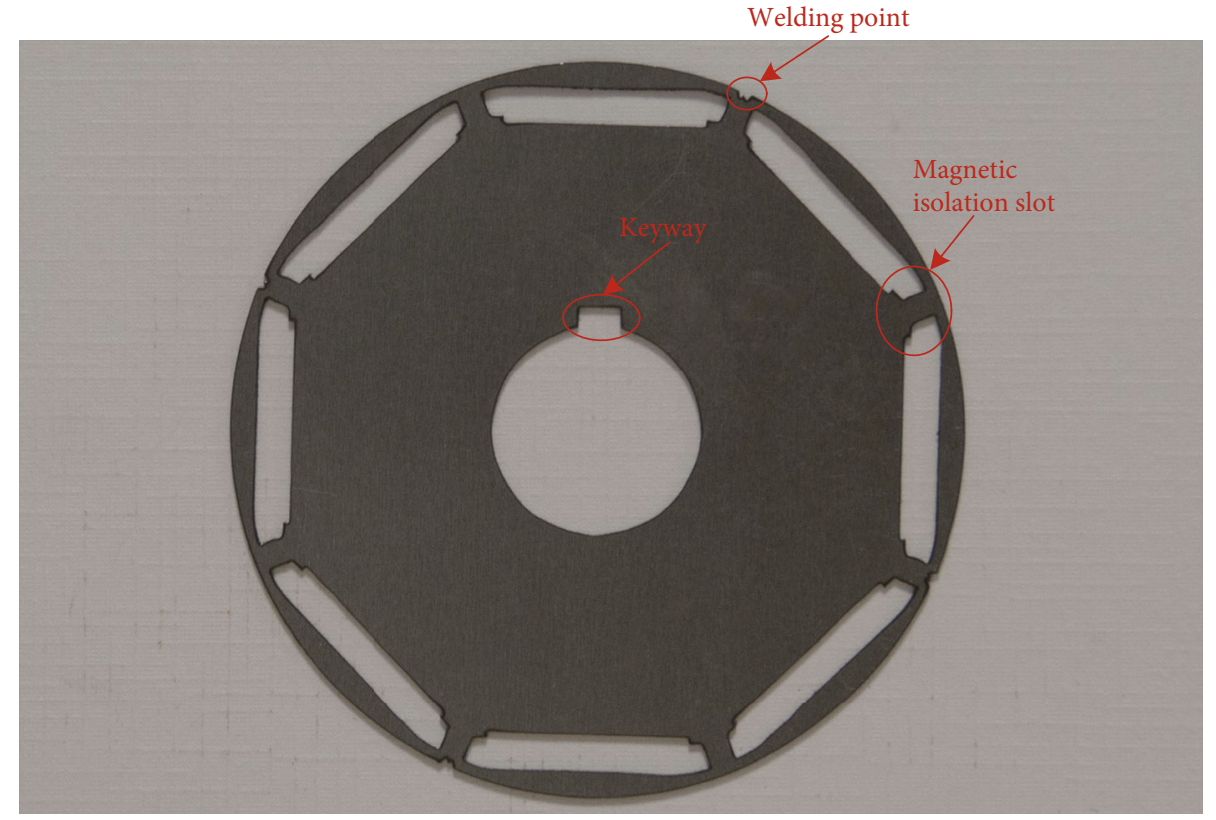

(b) The structure of rotor punching

FIGURE 1: The structure of the built-in radial PMG and the rotor punching.

the MMF, and the internal permeance of PMS. The magnetic circuit part of all these parameters can be simplified and equivalent to the rotor MMF, and the simplified equivalent magnetic circuit model is shown in Figure 4. Therefore, analyzing and improving the rotor MMF can effectively improve the main air-gap magnetic field and improve the output performance of the generator.

When the built-in radial PMG expands along the outer diameter of the rotor in the circumferential direction and ignores the influence of the stator slot, the distribution of the rotor MMF is shown in Figure 5.

In Figure 5, the coordinate axis $x$ is the circumferential length coordinate along the outer diameter of the rotor, the axis $y$ is the radial length coordinate of the generator, $F$ is the rotor MMF in the air gap, $F_{a}$ is the amplitude of the rotor MMF, $t_{p}$ is the effective calculation length of the PMS, and $t_{s}$ is the rotor pole moment. It can be seen from Figure 5 that the rotor MMF in the main air gap is approximately rectangular due to the leakage magnetic flux at the ends of the PMS and the magnetic flux loss of the rotor core. To simplify the analysis, the rotor MMF is equivalent to the standard rectangle, as shown in the lower coordinate system of Figure 5. Moreover, assuming that the axial direction is infinite and ignoring the axial length of the generator, the rotor MMF with circumferential distribution $F(x)$ can be calculated as follows: 


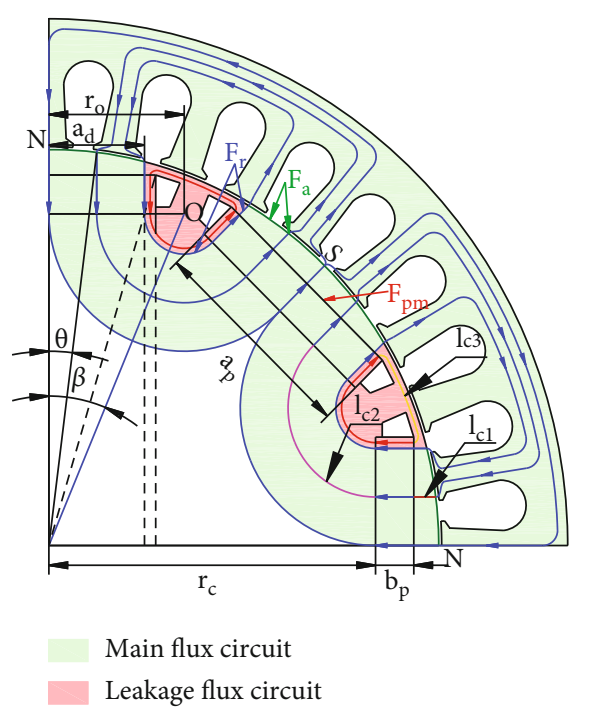

FIGURE 2: The magnetic circuit analysis diagram of generator.

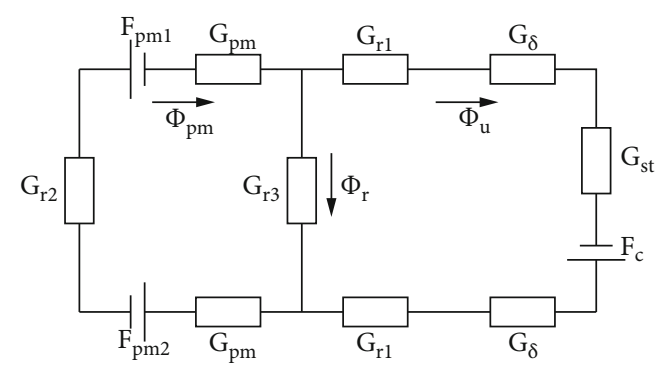

FIGURE 3: Equivalent magnetic circuit model of generator.

$$
F(x) \begin{cases}0, & k t_{s}-\frac{t_{s}-t_{p}}{2} \leq x<k t_{s}+\frac{t_{s}-t_{p}}{2}, \\ (-1)^{k} F_{a}, & k t_{s}+\frac{t_{s}-t_{p}}{2} \leq x<k t_{s}+\frac{t_{s}+t_{p}}{2} .\end{cases}
$$
follows:

The Fourier decomposition of $F(x)$ can be obtained as

$$
F(x)=\sum_{n=1}^{\infty}\left(\begin{array}{c}
(-1)^{(n+3) / 2} \frac{4 F_{a}}{n \pi} \\
\sin \left(\frac{n \pi t_{\mathrm{p}}}{2 t_{s}}\right) \sin \left(\frac{n \pi}{t_{s}} x\right)
\end{array}\right) n=1,3,5, \cdots
$$

where $n$ is the number of harmonics.

The amplitude of the rotor MMF $F_{a}$ is related to the magnetic circuit of the generator. For analytical calculation, the internal magnetic circuit of the rotor core is equivalent to a circle, as shown in Figure 2. And it can be seen from Figure 2 and the equivalent magnetic circuit model that the rotor MMF of each pole of the rotor is jointly provided by the N-pole and S-pole PMS and the MMF of two parts through the rotor core to the outer circular of the rotor. Therefore, the rotor MMF is the MMF generated by two

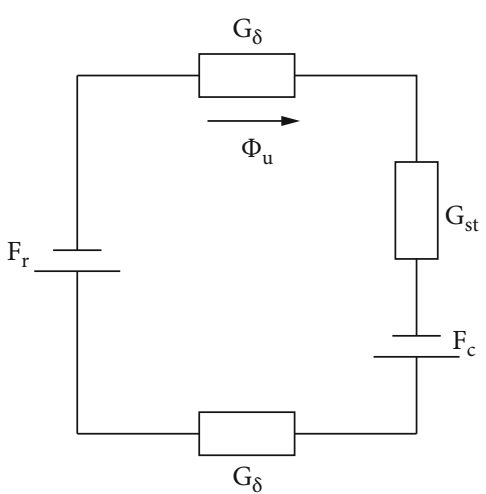

FIgURE 4: Simplified equivalent magnetic circuit model of generator.

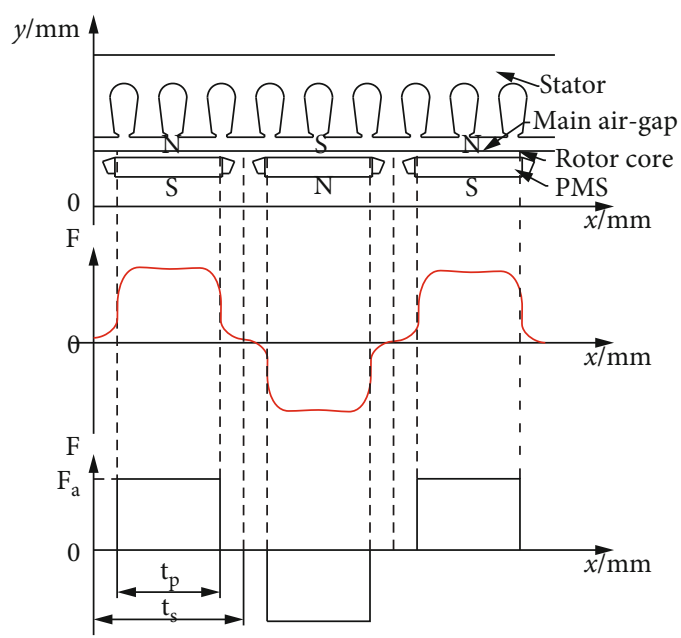

FIgURE 5: The circumferential distribution of rotor MMF.

PMSs minus the MMF of two parts of the rotor core. And $F_{a}$ can be calculated as follows:

$$
F_{a}=2 F_{\mathrm{pm}}-2 F_{r}
$$

where $F_{\mathrm{pm}}$ is the MMF on the surface of PMS and $F_{r}$ is the MMF when the MMF provided by PMS passes through the rotor core.

Considering the leakage magnetic flux at the ends of the PMS, the intersection point between the inner radial edge of the PMS and the connecting line between the top vertex of the end of the PMS and the rotor center is taken as the dividing point of the leakage magnetic flux and the main magnetic flux, and it can be seen in Figure 2. The part between the dividing points on both sides of PMS is recorded as the effective calculation length of PMS $t_{p}$. The arc length of the outer diameter of the rotor corresponding to the two dividing points is the effective calculation length of the rotor pole and is recorded as $t_{e}$. The relationship between them is as follows:

$$
t_{e}=r \arctan \frac{t_{p}}{2 r_{c}},
$$


TABLe 2: The main magnetic properties of PMS materials.

\begin{tabular}{lcccc}
\hline Name & Model number & $\begin{array}{c}\text { Magnetic induction } \\
\text { strength }(\mathrm{T})\end{array}$ & $\begin{array}{c}\text { Maximum coercivity } \\
(\mathrm{kA} / \mathrm{m})\end{array}$ & $\begin{array}{c}\text { Maximum magnetic energy } \\
\text { product }\left(\mathrm{kJ} / \mathrm{m}^{3}\right)\end{array}$ \\
\hline Ferrite PMS material & Y30 & 0.4 & 210 & 30 \\
Rare earth cobalt PMS material & XG196/96 & 0.96 & 690 & 183 \\
Nd-Fe-B PMS material & NdFe35 & 1.17 & 868 & 287 \\
\hline
\end{tabular}

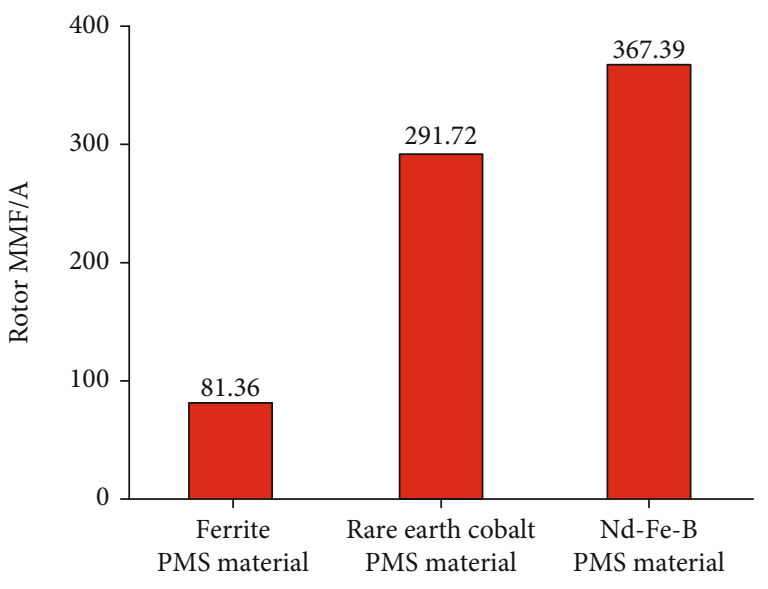

Different PMS materials

FIGURE 6: The rotor MMF histogram with different PMS materials.

where $r$ is the outer diameter of the rotor and $r_{c}$ is the distance from the center of the inner edge of PMS to the rotor center and is the vertical length of the inner side of PMS.

According to the geometric relationship, the radial effective calculation length of PMS $t_{p}$ can be calculated as follows:

$$
t_{p}=\frac{r_{c} a_{p}}{r_{c}+b_{p}}
$$

where $a_{p}$ is the radial length of PMS and $b_{p}$ is the thickness of PMS in magnetizing direction.

Taking one-fourth of the effective radial calculation length of the PMS as the equivalent calculation point of the rotor magnetic potential and according to the geometric relationship, the magnetic circuit length $l_{c 1}$ of the rotor core outside the PMS can be calculated as follows:

$$
l_{c 1}=r \sin \left(\arccos \frac{t_{p}}{4 r}\right)-r_{c}-b_{p}
$$

In Figure 2, the intersection $O$ of the tangential extension line in the inner side of PMS and the pole division line is taken as the center of the magnetic circuit of the rotor core in the inner side of PMS, and the distance from the center of the inner side of PMS to the center of the magnetic circuit $O$ is recorded as $r_{o}$; it can be calculated as

$$
r_{o}=r_{c} \tan \frac{\pi}{p}
$$

The length of magnetic circuit $l_{c 2}$ between the inner calculation points of N-pole PMS and S-pole PMS can be calculated as

$$
l_{c 2}=\frac{\pi}{4 p}(p-2)\left(4 r_{o}-t_{p}\right)
$$

Therefore, the MMF of rotor core $F_{r}$ can be calculated as

$$
F_{r}=\frac{B_{r}\left(l_{c 1}+l_{c 2}\right)}{\mu_{r}},
$$

where $B_{r}$ is the magnetic induction intensity of the rotor core, $\mu_{r}$ is the permeability of the rotor core, and $\mu_{r}=\mu_{r 0}$ $\mu_{0}$, where $\mu_{r 0}$ is the relative permeability of the rotor core, because the rotor core is made of silicon steel laminated, and $\mu_{r 0}=8000 \mathrm{H} \mathrm{m}^{-1}$, and $\mu_{0}$ is the vacuum permeability, and $\mu_{0}=4 \pi \times 10^{-7} \mathrm{H} \mathrm{m}^{-1}$.

The MMF of PMS $F_{p}$ can be calculated as follows:

$$
F_{p}=\frac{B_{p} b_{p}}{\mu_{p}}
$$

where $B_{p}$ is the magnetic induction intensity of the PMS, $\mu_{p}$ is the permeability of the PMS, and $\mu_{p}=\mu_{p 0} \mu_{0}$, where $\mu_{p 0}$ is the relative permeability of the rotor core; usually, $\mu_{p 0}$ is 1.05 1.1.

Taking the above formula into Equation (3), the amplitude of the rotor MMF $F_{a}$ can be calculated as follows:

$$
F_{a}=\frac{2 B_{p} b_{p}}{\mu_{p}}-\frac{2 B_{r}}{\mu_{r}}\left(\begin{array}{c}
r \sin \left(\arccos \frac{r_{c} a_{p}}{8 r\left(r_{c}+b_{p}\right)}\right)-r_{c}-b_{p} \\
+\frac{\pi}{4 p}(p-2)\left(2 r_{c} \tan \frac{\pi}{p}-\frac{r_{c} a_{p}}{2\left(r_{c}+b_{p}\right)}\right)
\end{array}\right) .
$$




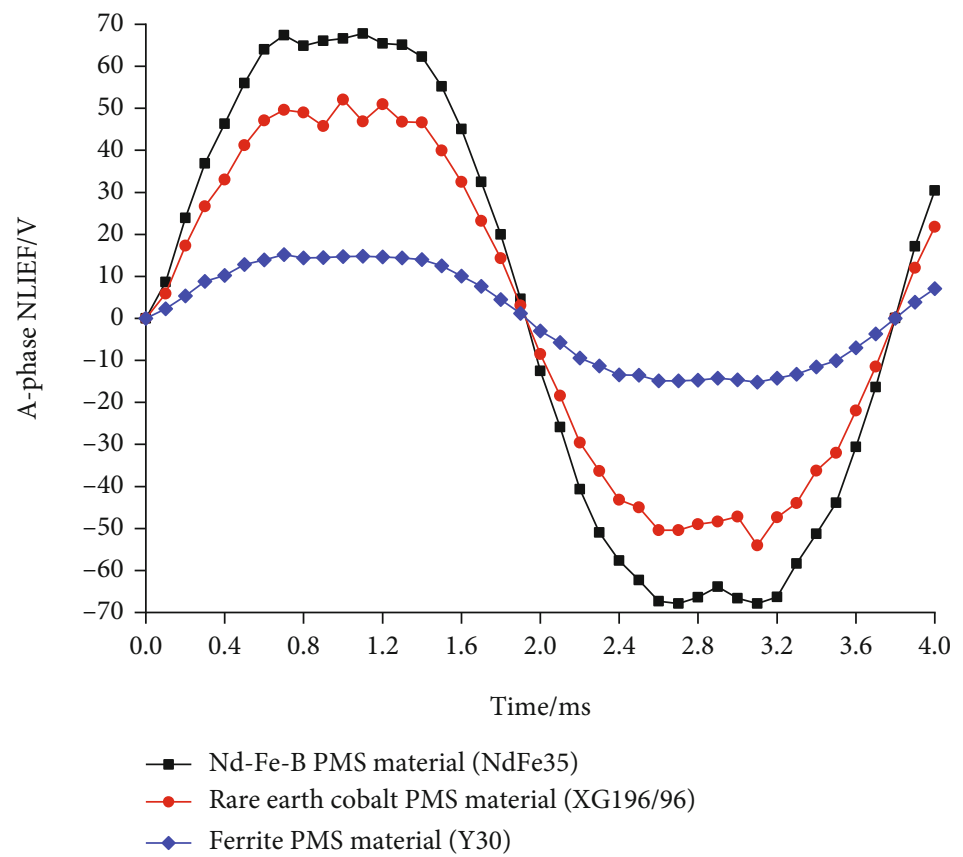

Figure 7: The NLIEF waveforms of different PMS materials.

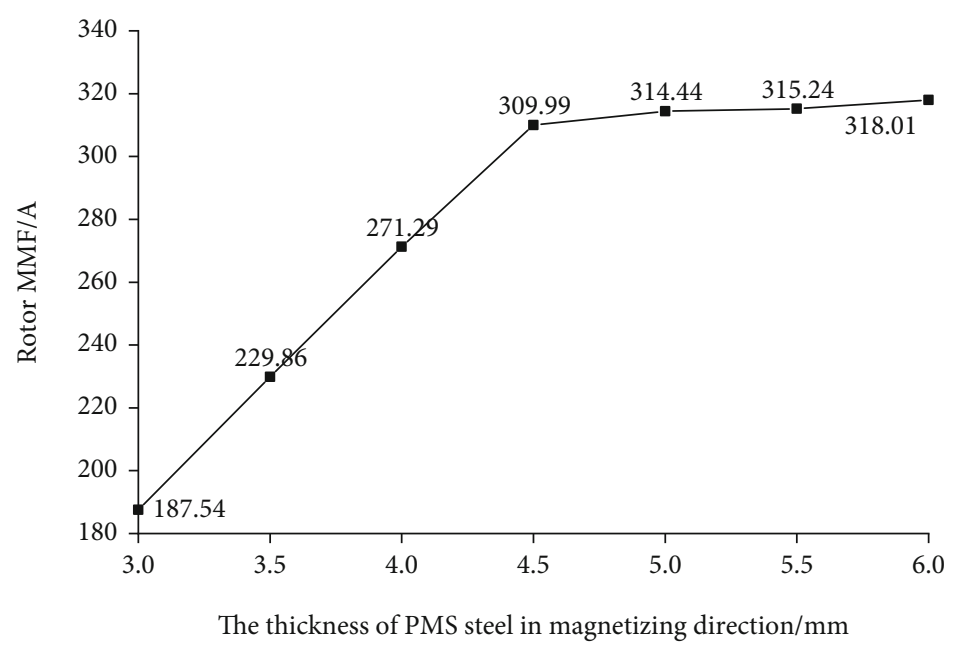

FIGURE 8: The rotor MMF curve with different thicknesses of PMS in magnetizing direction.

Taking Equation (11) into Equation (2), the Fourier decomposition of $F(x)$ is as follows:

$$
\left.F(x)=\sum_{n=1}^{\infty}\left(\begin{array}{c}
(-1)^{(n+3) / 2} \frac{4}{n \pi} \sin \left(\frac{n \pi t_{\mathrm{p}}}{2 t_{\mathrm{s}}}\right) \sin \left(\frac{n \pi}{t_{\mathrm{s}}} x\right) \\
\left.\frac{2 B_{p} b_{p}}{\mu_{p}}-\frac{2 B_{r}}{\mu_{r}}\left(\begin{array}{c}
r \sin \left(\arccos \frac{r_{c} a_{p}}{8 r\left(r_{c}+b_{p}\right)}\right)-r_{c}-b_{p} \\
+\frac{\pi}{4 p}(p-2)\left(2 r_{c} \tan \frac{\pi}{p}-\frac{r_{c} a_{p}}{2\left(r_{c}+b_{p}\right)}\right)
\end{array}\right)\right)
\end{array}\right)\right) n=1,3,5, \cdots
$$




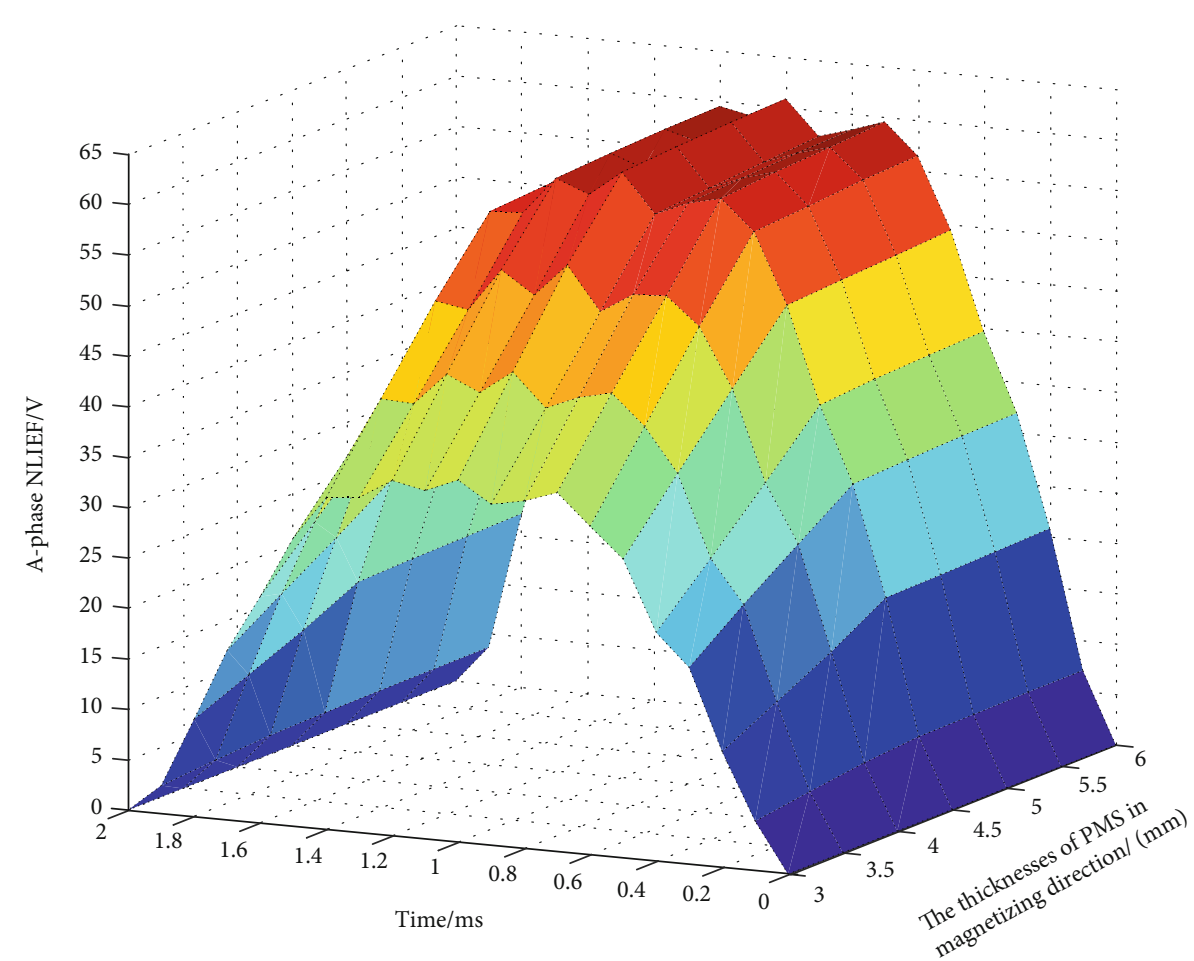

FIGURE 9: The NLIEF waveforms with different thicknesses of PMS in magnetizing direction.

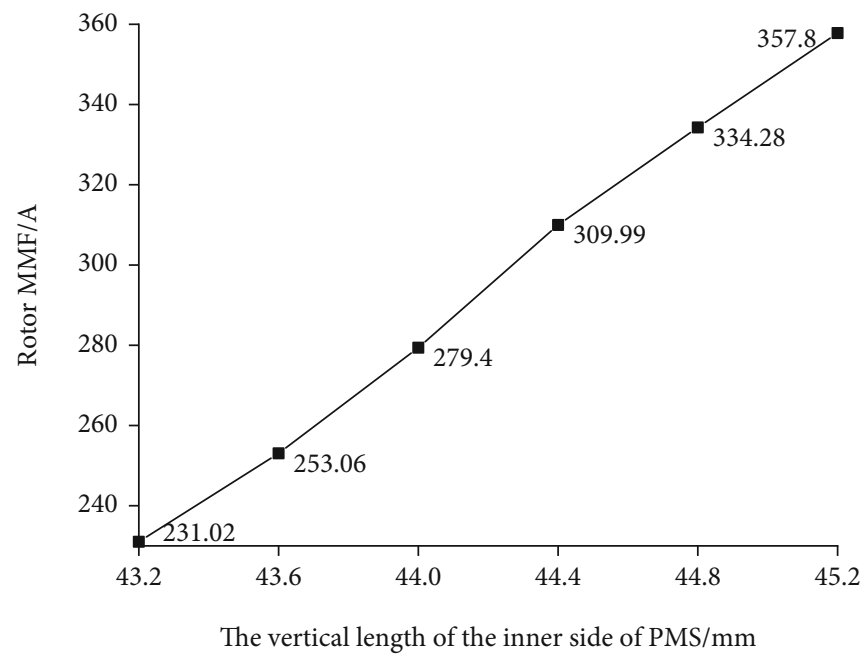

FIGURE 10: The rotor MMF curve with different vertical lengths of the inner side of PMS.

According to Equation (12), the main influencing parameters of the rotor MMF of the built-in radial PMG are the magnetic induction intensity of PMS $B_{p}$, the thickness of PMS in magnetizing direction $b_{p}$, the permeability of PMS $\mu_{p}$, the magnetic induction intensity of the rotor core $B_{r}$, the permeability of the rotor core $\mu_{r}$, the vertical length of the inner side of PMS $r_{c}$, the effective calculation length of PMS $t_{p}$, and the rotor pole moment $t_{s}$. Among them, the magnetic induction intensity and the permeability are the material parameters, and the rotor core of the generator is usually made of $0.5 \mathrm{~mm}$ silicon steel sheet; therefore, only the PMS material is considered. The rotor polar moment is mainly determined by the overall structure of the generator and is not considered temporarily. In conclusion, without considering the overall structural parameters of the generator and the rotor core material, the rotor MMF is mainly affected by the rotor parameters of the PMS materials, $h_{p}, r_{c}$, and $t_{p}$.

\section{Influencing Rules of Rotor Parameters on Rotor MMF}

3.1. The PMS Materials. There are many kinds of PMS materials used in vehicle generators, and the properties are quite different. Presently, three types of PMS materials are widely 


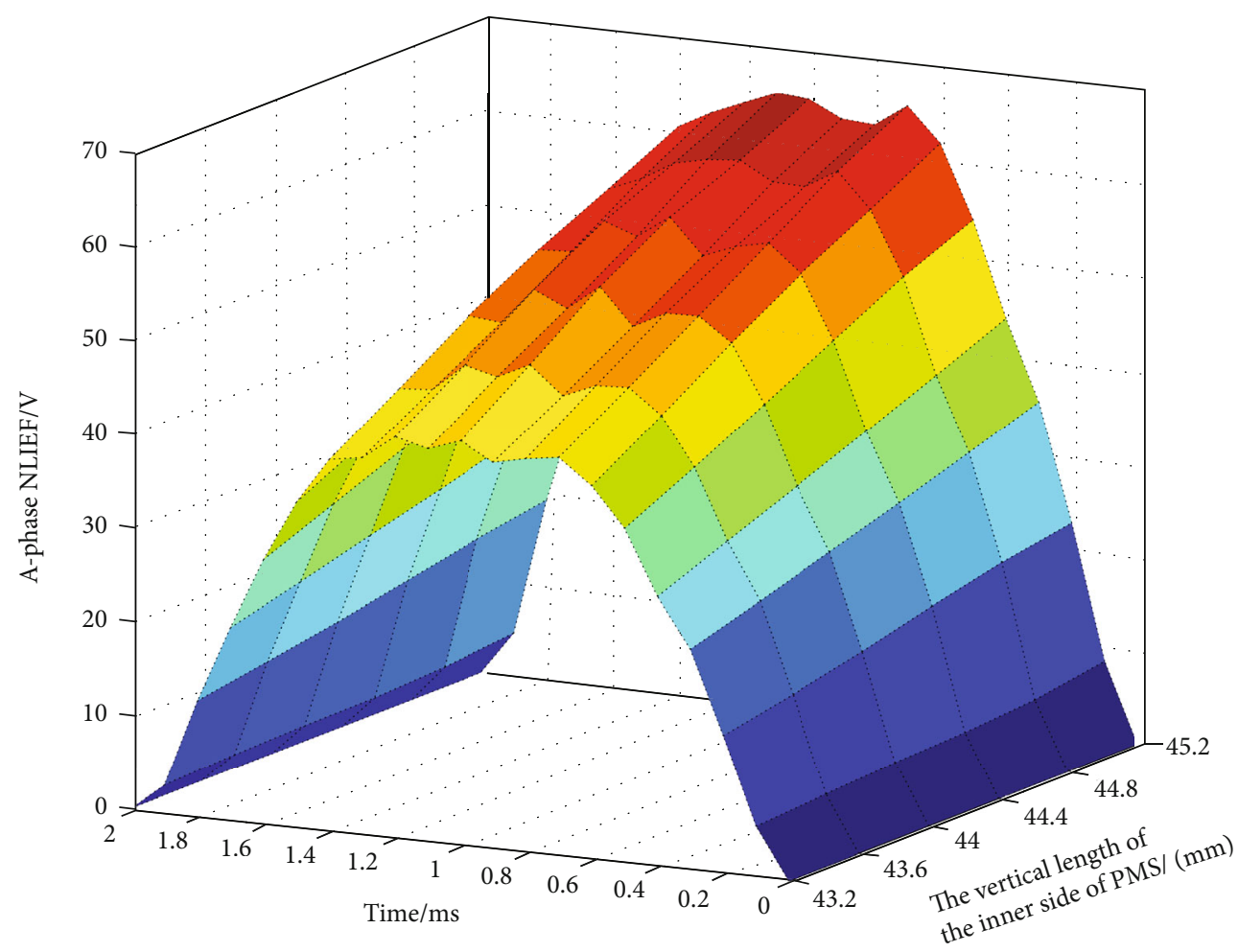

FIgURE 11: The NLIEF waveforms with different vertical lengths of the inner side of PMS.

used: the ferrite PMS material, the rare earth cobalt PMS material, and the neodymium iron boron (Nd-Fe-B) PMS material [23]. Their main magnetic properties are shown in Table 2.

Taking a 3-phase 8-pole 36-slot built-in radial PMG as an example, the outer diameter of the stator is $140 \mathrm{~mm}$, the inner diameter of the stator is $105 \mathrm{~mm}$, the axial length of the stator is $30 \mathrm{~mm}$, the main air-gap length is $0.4 \mathrm{~mm}$, and the inner diameter of the rotor is $20 \mathrm{~mm}$. Using the three kinds of PMS materials, the generator simulation model is established and simulated. The rotor MMF is calculated as the product of the rotor magnetic flux of each pole and the reluctance of the main magnetic circuit of the rotor. The rotor magnetic flux of each pole can be calculated by the field processor of the finite element software. The calculated results of rotor MMF with different PMS materials are shown in Figure 6, and the no-load induction electromotive force (NLIEF) waveforms are shown in Figure 7.

Figure 6 shows that the rotor MMFs with different PMS materials are quite different. Among them, the maximum value is $367.39 \mathrm{~A}$, which uses the Nd-Fe-B PMS material. A little lower is rare earth cobalt PMS material. And the minimum value is $81.36 \mathrm{~A}$, which uses the ferrite PMS material. It can also be seen from Figure 7 that the maximum value of NLIEF produced by rare earth cobalt PMS material can reach $50 \mathrm{~V}$, which can meet the requirement of rated voltage $14 \mathrm{~V}$. Meanwhile, rare earth cobalt PMS material is cheaper than Nd-Fe-B PMS material and therefore becomes the best PMS material in this paper.

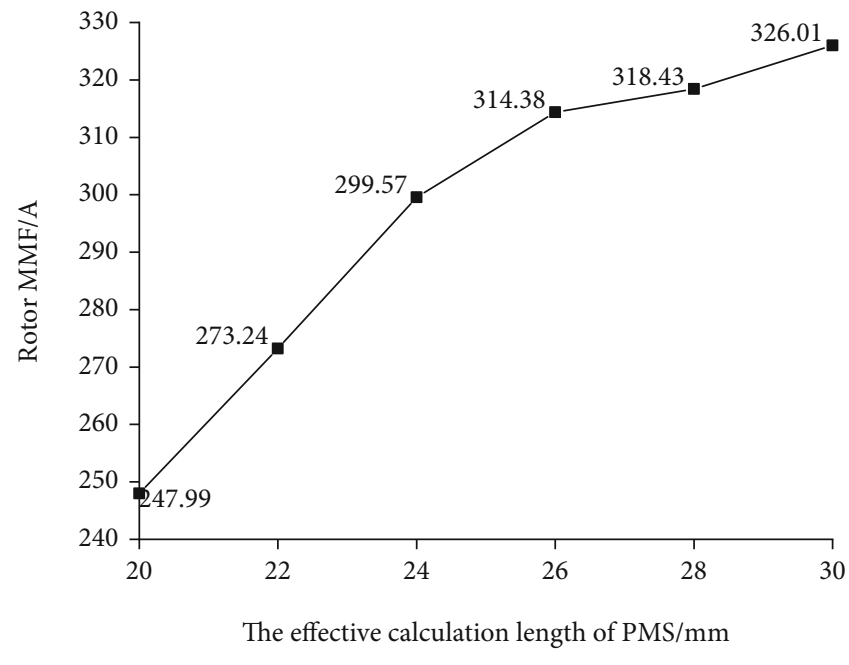

FIgURE 12: The rotor MMF curve with different effective calculation lengths of PMS.

3.2. The Thickness of PMS in Magnetizing Direction $h_{p}$. Using the finite element model, change the thickness of PMS in magnetizing direction $h_{p}$ from $3 \mathrm{~mm}$ to $6 \mathrm{~mm}$ in steps of $0.5 \mathrm{~mm}$. The variation diagrams of the rotor MMF and the NLIEF waveform are simulated as shown in Figures 8 and 9 , respectively.

Figure 8 shows that with the increase of the thickness of PMS in magnetizing direction $h_{p}$, the rotor MMF increases continuously, but the increase decreases when $h_{p}$ increases to $4.5 \mathrm{~mm}$; it can also be seen in Figure 9 , when $h_{p}$ is from 


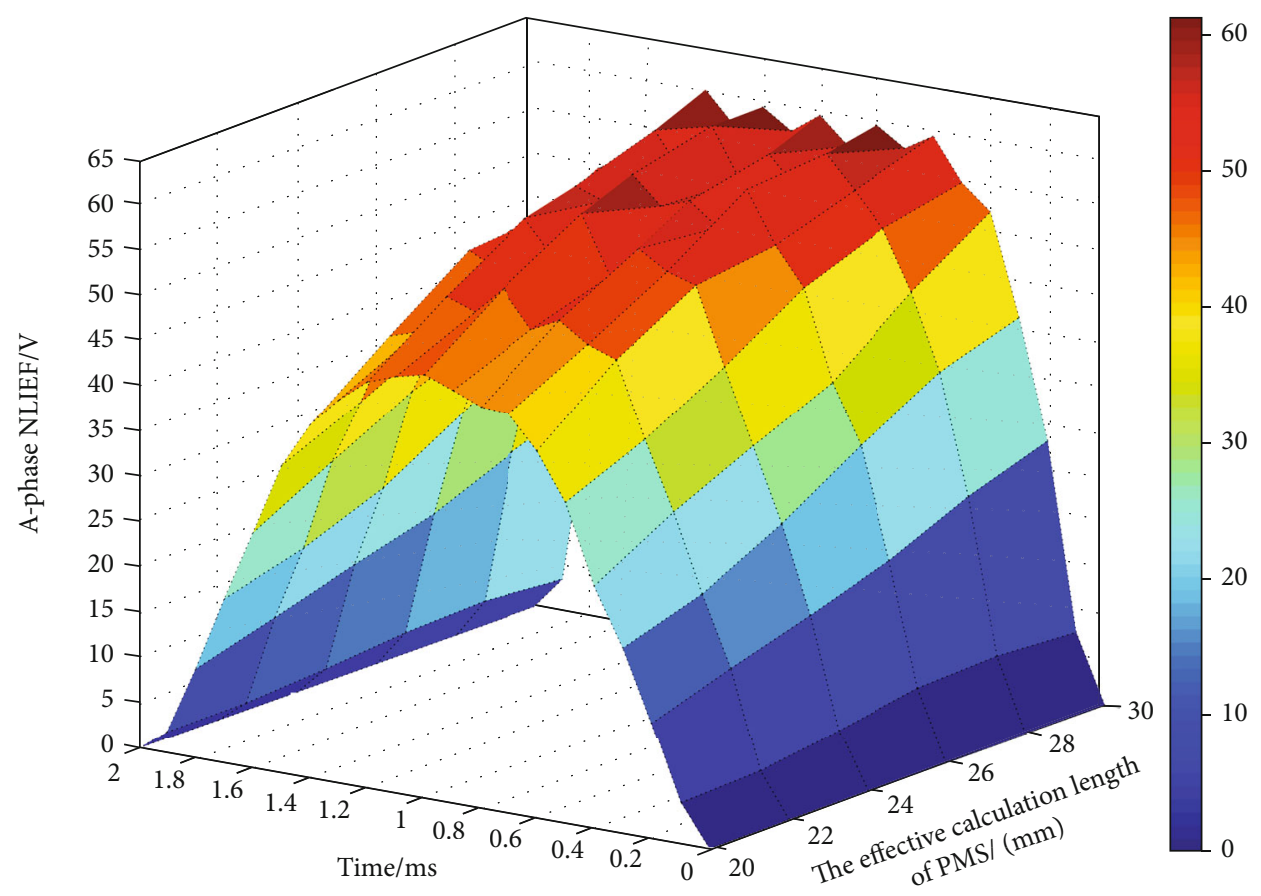

FIgURE 13: The NLIEF waveforms with different effective calculation lengths of PMS.

$4.5 \mathrm{~mm}$ to $6 \mathrm{~mm}$, the NLIEF waveforms change very little. Therefore, considering the rotor MMF and material utilization ratio, it is more appropriate to select the thickness of PMS in magnetizing direction from $4 \mathrm{~mm}$ to $4.5 \mathrm{~mm}$.

3.3. The Vertical Length of the Inner Side of PMS $r_{c}$. When the vertical length of the inner side of PMS $r_{c}$ is from $43.2 \mathrm{~mm}$ to $45.2 \mathrm{~mm}$ and the step is $0.5 \mathrm{~mm}$, the simulation results of the rotor MMF curve and the NLIEF waveform are simulated and shown in Figures 10 and 11, respectively.

As shown in Figures 10 and 11, with the increase of vertical length of the inner side of PMS, the rotor MMF and the peak of NLIEF waveform increase. However, if $r_{c}$ is too large, the PMS will be too close to the outer circle of the rotor, and the structural strength of the rotor will be reduced, whereas if $r_{c}$ is too small, the leakage magnetic flux at the ends of the PMS will increase, and the rotor MMF will decrease. When $r_{c}$ is from $44 \mathrm{~mm}$ to $44.4 \mathrm{~mm}$, the rotor MMF is from $279.4 \mathrm{~A}$ to $309.99 \mathrm{~A}$, and the peak value of NLIEF is about $55 \mathrm{~V}$, which can meet the output requirements. Therefore, it is more appropriate to take $r_{c}$ from $44 \mathrm{~mm}$ to $44.4 \mathrm{~mm}$.

3.4. The Effective Calculation Length of PMS $t_{p}$. When the effective calculation length of PMS $t_{p}$ is from $20 \mathrm{~mm}$ to $30 \mathrm{~mm}$ and the step is $2 \mathrm{~mm}$, the simulation results of the rotor MMF curve and the NLIEF waveform are shown in Figures 12 and 13, respectively.

As shown in Figures 12 and 13, with the increase of the effective calculation length of PMS $t_{p}$, the rotor MMF increases, and the peak value of the NLIEF waveform increases too, whereas when $t_{p}$ increases to $26 \mathrm{~mm}$, the increasing trend of rotor MMF and the NLIEF becomes
TABLE 3: Grouping table of optimization parameters.

\begin{tabular}{lccccccc}
\hline $\begin{array}{l}\text { Group } \\
\text { number }\end{array}$ & $\begin{array}{c}h_{p} \\
(\mathrm{~mm})\end{array}$ & $\begin{array}{c}r_{c} \\
(\mathrm{~mm})\end{array}$ & $\begin{array}{c}t_{p} \\
(\mathrm{~mm})\end{array}$ & $\begin{array}{c}\text { Group } \\
\text { number }\end{array}$ & $\begin{array}{c}h_{p} \\
(\mathrm{~mm})\end{array}$ & $\begin{array}{c}r_{c} \\
(\mathrm{~mm})\end{array}$ & $\begin{array}{c}t_{p} \\
(\mathrm{~mm})\end{array}$ \\
\hline 1 & 4 & 44 & 24 & 15 & 4.3 & 44.2 & 26 \\
2 & 4 & 44 & 25 & 16 & 4.3 & 44.4 & 24 \\
3 & 4 & 44 & 26 & 17 & 4.3 & 44.4 & 25 \\
4 & 4 & 44.2 & 24 & 18 & 4.3 & 44.4 & 26 \\
5 & 4 & 44.2 & 25 & 19 & 4.6 & 44 & 24 \\
6 & 4 & 44.2 & 26 & 20 & 4.6 & 44 & 25 \\
7 & 4 & 44.4 & 24 & 21 & 4.6 & 44 & 26 \\
8 & 4 & 44.4 & 25 & 22 & 4.6 & 44.2 & 24 \\
9 & 4 & 44.4 & 26 & 23 & 4.6 & 44.2 & 25 \\
10 & 4.3 & 44 & 24 & 24 & 4.6 & 44.2 & 26 \\
11 & 4.3 & 44 & 25 & 25 & 4.6 & 44.4 & 24 \\
12 & 4.3 & 44 & 26 & 26 & 4.6 & 44.4 & 25 \\
13 & 4.3 & 44.2 & 24 & 27 & 4.6 & 44.4 & 26 \\
14 & 4.3 & 44.2 & 25 & & & & \\
\hline
\end{tabular}

slower. And with the increase of $t_{p}$, the consumption of PMS also increases. So comparing comprehensively, it is more appropriate to select $t_{p}$ from $24 \mathrm{~mm}$ to $26 \mathrm{~mm}$.

3.5. Multiparameter Optimization Analysis. Taking the thickness of PMS in magnetizing direction, the vertical length of the inner side of PMS, and the effective calculation length of PMS as optimization parameters, the multiparameter optimization analysis of rotor MMF is carried out by finite element software. Each parameter analysis value is set up to refer to the above optimization results, and all parameters are subdivided into 27 groups, as shown in Table 3. 


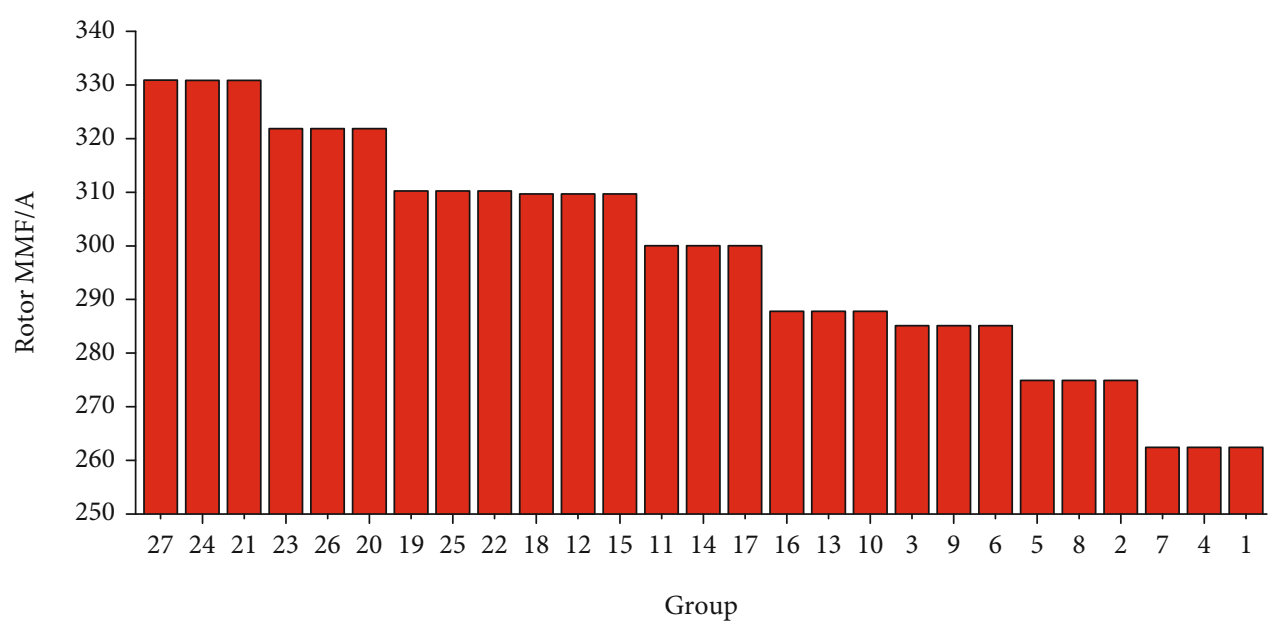

FIGURE 14: The rotor MMF change histogram of different combinations of rotor parameters.

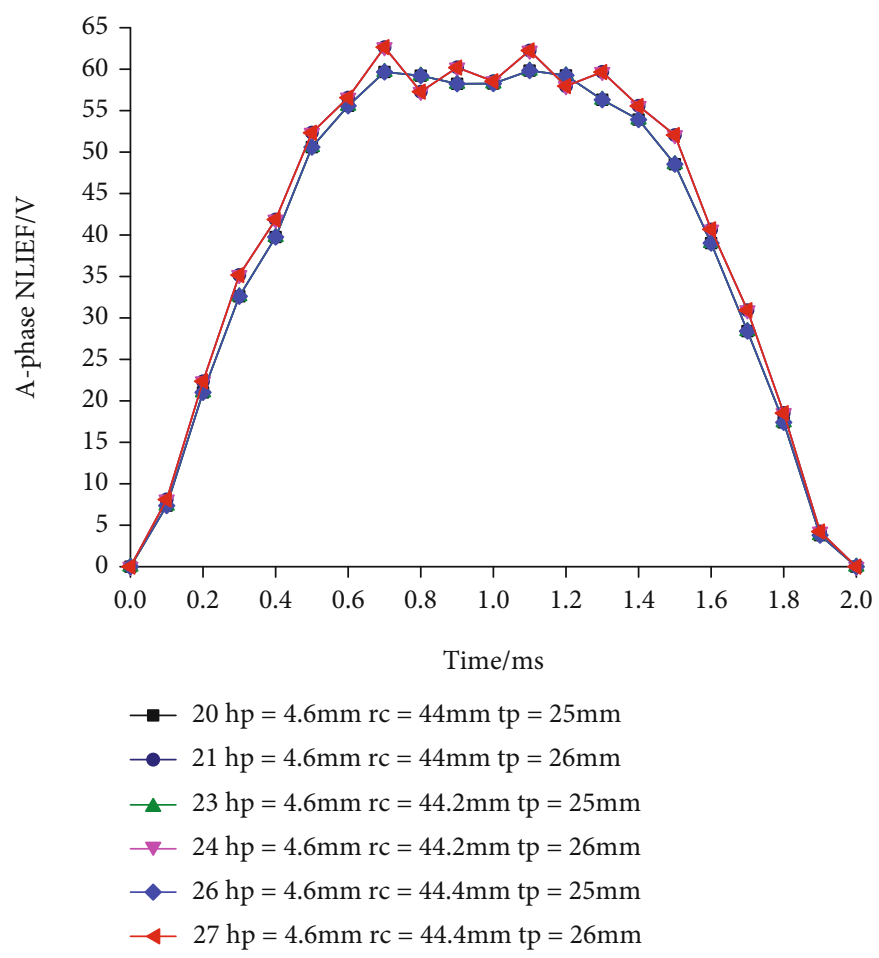

FIGURE 15: The NLIEF waveforms of different combinations of rotor parameters.

TABLE 4: The optimized rotor pole parameters.

\begin{tabular}{lc}
\hline Main parameters & Parameter values \\
\hline PMS material & Rare earth cobalt PMS material (XG196/96) \\
The thickness of PMS in magnetizing direction $h_{p}$ & $4.6 \mathrm{~mm}$ \\
The vertical length of the inner side of PMS $r_{c}$ & $3.6 \mathrm{~mm}$ \\
The effective calculation length of PMS $t_{p}$ & $25 \mathrm{~mm}$ \\
\hline
\end{tabular}




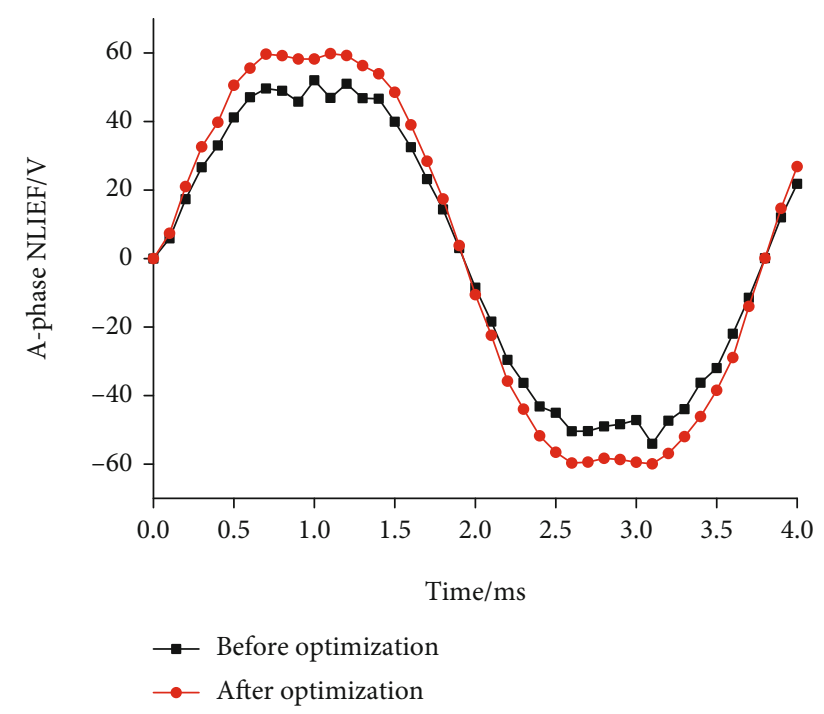

FIgURE 16: The NLIEF waveform before and after optimization.

Three parameters with different values are input into finite element software for parametric simulation, the rotor MMFs of different groups are obtained, and its histogram is drawn in order from big to small, as shown in Figure 14.

Figure 14 shows that the rotor MMF varies with the different combinations of rotor parameters. And the first six groups of high rotor MMF, including group 20, group 21, group 23, group 24, group 26, and group 27, are selected to analyze the NLIEF waveform as shown in Figure 15.

Figure 15 shows that the NLIEF waveforms of group 21, group 24, and group 27 almost overlap, as well as group 20, group 23, and group 26. The peaks of NLIEF waveforms in the second overlap group are slightly lower than those in the first overlap grouping, but the smoothness of the curve increases, the distortion rate at the top of the peak decreases, and the sinusoidal property of the whole curve increases. Therefore, group 20, group 23, and group 26 have higher application values. And the data analysis of these three groups shows that only the vertical lengths of the inner side of PMS are different, which are $44 \mathrm{~mm}, 44.2 \mathrm{~mm}$, and $44.4 \mathrm{~mm}$, respectively. Because the larger the vertical length of the inner side of PMS, the closer the PMS is to the outer circle of the rotor and the lower the structural strength of the rotor. Therefore, the design parameters of group 26 are selected as the optimal design parameters.

\section{The Analysis of Optimization Results}

After optimization and analysis, the optimized rotor pole parameters are shown in Table 4 . The finite element simulation model is established and simulated by using the optimized parameters, and the comparison of the NLIEF curves before and after optimization is shown in Figure 16.

Figure 16 shows that the peak value of the NLIEF waveform of the optimized PMG is obviously improved, the smoothness of the peak top is improved, and the sinusoidal property is enhanced. The rotor MMFs before and after optimization are 291.72 A and 321.84 A, respectively, which

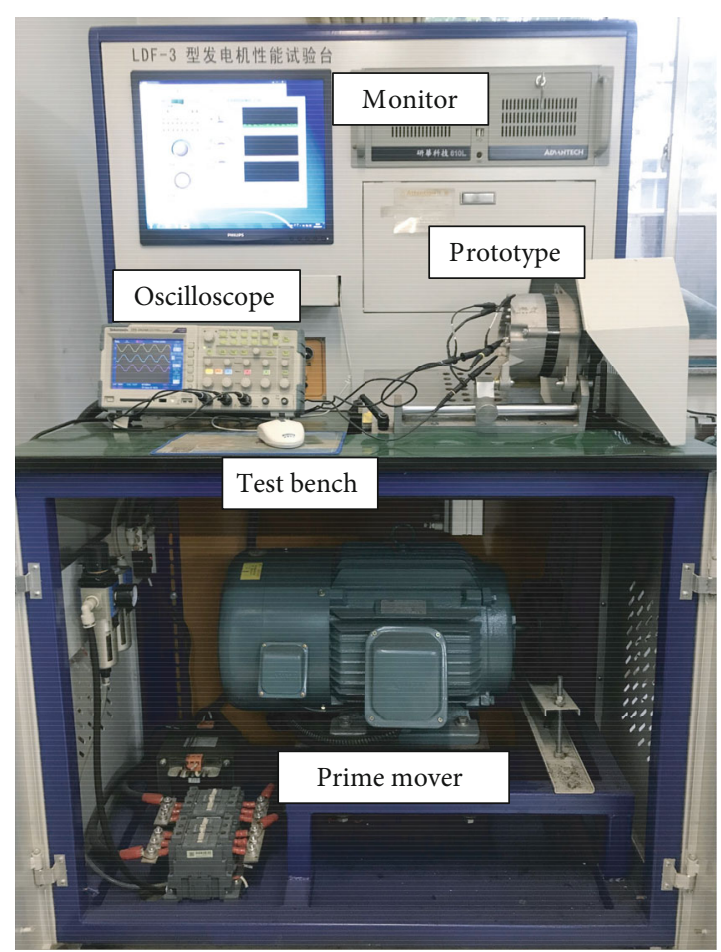

FIGURE 17: The test bench.

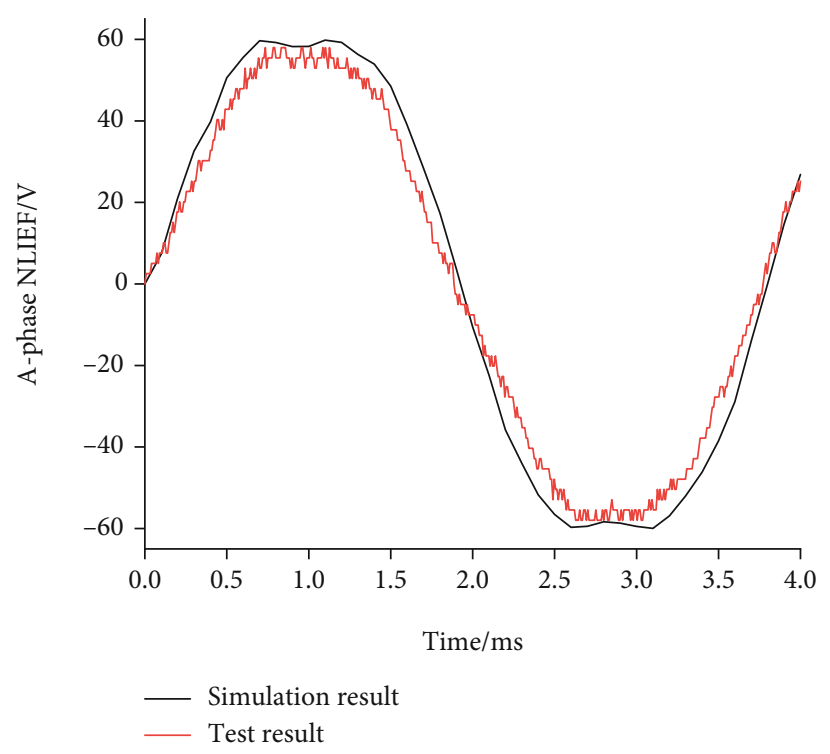

FIgURE 18: The NLIEF waveform obtained by the test and the finite element simulation.

increases by $10.3 \%$ after optimization. This will effectively increase the magnetic flux density in the main air gap and improve the output performance of the generator.

To verify the effectiveness of finite element simulation, the prototype is trial produced with the optimized parameters, and the bench test is carried out with the generator performance test bench. The test bench is shown in Figure 17, and the comparison of NLIEF waveforms obtained by the test and the finite element simulation is shown in Figure 18. 
It can be seen from Figure 18 that the peak value of the NLIEF waveform in the test is slightly lower than the simulation result, but the NLIEF waveform is basically the same. Therefore, the finite element analysis result is effective.

\section{Conclusions}

In this paper, the analytical expression of the rotor MMF of the built-in radial PMG is derived in detail, and the main influence parameters are analyzed. Moreover, the variation rules of rotor MMF with the PMS material, the thickness of PMS in magnetizing direction, the vertical length of the inner side of PMS, and the effective calculation length of PMS are analyzed to optimize the rotor parameters of a 3phase 8-pole 36-slot built-in radial PMG. After optimization, the rotor MMF increases by $10.3 \%$, the main air-gap flux density increases significantly, the sinusoidal character of NLIEF becomes better, and the output characteristics of the generator improve obviously.

\section{Data Availability}

All data used to support the findings of this study are available from the corresponding author upon request.

\section{Conflicts of Interest}

The authors declare that there is no conflict of interest regarding the publication of this paper.

\section{Acknowledgments}

This research work is partially supported by the National Natural Science Foundation of China (Grant Nos. 518753270 and 51975340).

\section{References}

[1] Y.Z. Hua, H. Q. Zhu, and Y. Xu, "Multi-objective optimization design of bearingless permanent magnet synchronous generator," IEEE Transactions on Applied Superconductivity, vol. 30, no. 4, pp. 591-600, 2020.

[2] D. K. Lim, S. Y. Jung, K. P. Yi, and H. K. Jung, "A novel sequential-stage optimization strategy for an interior permanent magnet synchronous generator design," IEEE Transactions on Industrial Electronics, vol. 65, no. 2, pp. 1781-1790, 2017.

[3] A. S. Aladsani and O. Beik, "Design of a multiphase hybrid permanent magnet generator for series hybrid EV," IEEE Transactions on Energy Conversion, vol. 33, no. 3, pp. 1-9, 2018.

[4] S. Wu, T. Shi, L. Guo, H. Wang, and C. Xia, "Accurate analytical method for magnetic field calculation of interior PM motors," IEEE Transactions on Energy Conversion, vol. 99, pp. 1-12, 2021.

[5] S. Chen, Q. Zhang, S. Huang, and J. Guo, "Multiphysicaldomain integration design of oil-cooled permanent magnet ISG motor," Mathematical Problems in Engineering, vol. 2020, Article ID 2818403, 10 pages, 2020.

[6] W. Sriwannarat, A. Siritaratiwat, and P. Khunkitti, "Structural design of partitioned stator doubly salient permanent magnet generator for power output improvement," Advances in Materials Science and Engineering, vol. 2019, Article ID 2189761, 8 pages, 2019.

[7] S. Wu, Q. Chen, Q. Li, X. Liu, H. Zhang, and L. Lin, "Design of aviation high impedance permanent magnet synchronous generator," Mathematical Problems in Engineering, vol. 2021, 10 pages, 2010.

[8] R. E. Quintal-Palomo, M. G. Wozdziewicz, and M. Dybkowski, "Modelling and co-simulation of a permanent magnet synchronous generator," COMPEL, vol. 38, no. 6, pp. 19041917, 2019.

[9] Q. F. Lin, S. X. Niu, F. B. Cai et al., "Design and optimization of a novel dual-PM machine for electric vehicle applications," IEEE Transactions on Vehicular Technology, vol. 69, no. 12, pp. 14391-14400, 2020.

[10] Q. Tan, X. Huang, L. Li, and M. Wang, "Magnetic field analysis and flux barrier design for modular permanent magnet linear synchronous motor," IEEE Transactions on Industrial Electronics, vol. 67, no. 5, pp. 3891-3900, 2020.

[11] A. Ck, B. Aka, and A. Wk, "Modeling of a permanent magnet linear synchronous motor using magnetic equivalent circuits," Mechatronics, vol. 76, pp. 1-9, 2021.

[12] P. Vahedi and B. Ganji, "A simple magnetic equivalent circuit model for switched reluctance machine," Electrical Engineering, vol. 103, no. 11, pp. 1-14, 2021.

[13] S. Y. Qin, G. Y. Xiong, and H. Niu, "Analysis of magnetic field in air gap due to permanent magnet motor," Journal of Taiyuan University of Technology, vol. 33, no. 2, pp. 121-124, 2002.

[14] S. T. Boroujeni, "Complex vector modeling of a doubly fed cascaded cage rotor induction machine," Electrical Engineering, vol. 102, no. 3, pp. 1831-1842, 2020.

[15] S. Zhu, Y. Hu, C. Liu, K. Wang, and W. Chen, "Reduction of stator core loss in interior PM machines for electric vehicle applications," IEEE Transactions on Magnetics, vol. 54, no. 11, pp. 1-5, 2018.

[16] W. Hu, X. Zhang, H. Yin, H. Geng, Y. Zhang, and L. Shi, "Analysis of magnetic field and electromagnetic performance of a new hybrid excitation synchronous motor with dual-V type magnets," Energies, vol. 13, no. 6, pp. 1-19, 2020.

[17] W. Zhang, L. Shi, K. Liu, L. Li, and J. Jing, "Optimization analysis of automotive asymmetric magnetic pole permanent magnet motor by Taguchi method," International Journal of Rotating Machinery, vol. 2021, Article ID 6691574, 2021.

[18] S. R. Karimpour, M. R. Besmi, and S. M. Mirimani, “Optimal design and verification of interior permanent magnet synchronous generator based on FEA and Taguchi method," International Transactions on Electrical Energy Systems, vol. 30, no. 11, pp. 1-15, 2020.

[19] H. Qiu, X. Zhao, Y. Wei, R. Yi, and C. Yang, "Influence of rectifier characteristic harmonics on loss and temperature field of high-speed permanent magnet generator," International Transactions on Electrical Energy Systems, vol. 30, no. 7, 2020.

[20] H. Y. Tang, W. X. Zhao, and C. Y. Gu, “A new adaptive control for five-phase fault-tolerant flux-switching permanent magnet motor," International Journal of Rotating Machinery, vol. 2016, Article ID 3792645, 15 pages, 2016.

[21] Y. P. Yang and C. J. Wu, "Design of a flux-shunt permanent magnet DC motor with hybrid magnetomotive force and 
anti-demagnetization property," IEEE Transactions on Magnetics, vol. 46, no. 11, pp. 3920-3927, 2010.

[22] Y. P. Zhang, Y. M. Wang, and S. N. Gao, “3-D magnetic equivalent circuit model for a coreless axial flux permanent-magnet synchronous generator," IET Electric Power Applications, vol. 46, no. 11, pp. 3920-3927, 2010.

[23] X. Zhang, Q. Du, S. Ma et al., "Permeance analysis and calculation of the double-radial rare-earth permanent magnet voltage-stabilizing generation device," IEEE Access, vol. 6, pp. 23939-23947, 2018. 